\title{
Characterisation of drug resistance gene cassettes associated with class 1 integrons in clinical isolates of Escherichia coli from Taiwan, ROC
}

\author{
CHUNG-YU CHANG, LIN-LI CHANG, YU-HUNG CHANG, TSONG-MING LEE* and \\ SHUI-FENG CHANG
} Department of Microbiology, School of Medicine, Kaohsiung Medical University, Kaohsiung 807 and
* Department of Food Sanitation, Tajen Institute of Technology, Ping Tung 907, Taiwan, ROC

\begin{abstract}
The presence of class 1 integrons in clinical isolates of Escherichia coli was detected by PCR. Of $104 E$. coli isolates from Kaohsiung, 54 (52\%) carried class 1 integrons, with inserted DNA regions of 1-3 kb. These integrons were located on plasmids, as demonstrated by Southern hybridisation. DNA sequencing was used to identify the genetic content of the integron-variable regions. Different class 1 integrons contained various numbers, kinds and combinations of gene cassettes within their variable regions. These gene cassettes included those encoding resistance to trimethoprim (dfrIa, dfrV, dfr12 and dfr17), aminoglycosides (aadA1a, aadA2, aadA4 and aadB), chloramphenicol $(\mathrm{cml} A)$, erythromycin (ere $A 2)$ and $\beta$-lactams (blaP1). An integron carrying three inserted cassettes - dfr12-orfF-aadA2 - was present in $33(61 \%)$ of the 54 isolates with class 1 integrons. Gene cassettes encoding resistance were expressed phenotypically. The results indicate that class 1 integrons are widespread in clinical $E$. coli isolates in Taiwan. The types, combinations and frequency of the gene cassettes in integrons may reflect the specific selective pressures to which the isolates were exposed and could provide useful surveillance data for relation to antibiotic usage information.
\end{abstract}

\section{Introduction}

The rapid dissemination of drug-resistant bacteria is an alarming and increasing problem, complicating the treatment of infections. Much of the problem is the result of antibiotic resistance genes transferring among bacterial species, carried by plasmids and transposons [1]. In recent years, a third mechanism for the dissemination of antibiotic resistance genes has been discovered; this involves integrons, novel DNA elements that mediate the integration of antibiotic resistance genes through site-specific recombination [1-3]. This recombination system depends on two determinants: an int gene encoding a site-specific DNA integrase (Int) [1,2], and an adjacent site, attI, which is recognised by the integrase and acts as the receptor for the inserted genes [3]. The inserted genes, which mostly encode antibiotic resistances, are contained in mobile elements called gene cassettes [4]. Each gene cassette consists of a coding region and an integrase-

Received 18 Feb. 2000; revised version accepted 26 April 2000.

Corresponding author: Dr S-F. Chang (e-mail: shfech@cc. kmu.edu.tw). specific recombination site known as a 59-base element $[4,5]$. Most of the gene cassettes lack their own promoters, and integrons act as natural expression vectors by supplying a common promoter, $\mathrm{P}_{\text {ant }}$, located in the conserved sequences upstream of the inserted genes [2-4].

Four types of integrons, with different int genes, have been identified. Most integrons from clinical isolates belong to class 1 [6] and consist of two conserved segments. A 5'-conserved segment contains the int gene (intI1), the attI site and the common promoters $\mathrm{P}_{\text {ant }}[3,4]$, whereas the 3 '-conserved segment includes an antiseptic resistance gene (qacE $\Delta l)$, a sulphonamide resistance gene (sull) and an open reading frame (orf5) of unknown function [2]. The central variable region includes different combinations of inserted gene cassettes. More than 40 different antibiotic resistance cassettes have been characterised from integrons, which consequently can allow their bacterial hosts to become resistant to broad spectra of antimicrobial agents [4].

Several studies have investigated the prevalence of integrons in clinical isolates and have found them to be widespread [7-9]. However, the kinds of gene cassettes 
inserted into the integrons were not identified, except in the work of Sallen et al. [7]. In Taiwan, resistance to various antibiotics is very common in clinical isolates, often more so than in Western countries [10]. However, the frequency, characteristics, and roles of integrons and gene cassettes have not yet been investigated. In this study, Escherichia coli isolates from Kaohsiung, Taiwan, were investigated to demonstrate the prevalence and characteristics of class 1 integrons and the gene cassettes in these organisms, relating the results to the phenotypic expression of resistance.

\section{Materials and methods}

\section{Bacterial strains}

A total of 104 E. coli isolates was collected consecutively in 1993 and 1994 from the Kaohsiung Medical University Hospital, a medical centre in southern Taiwan. They were isolated from various clinical specimens from diverse units of the hospital. Once identified, the isolates were preserved at $-70^{\circ} \mathrm{C}$ in Tryptic Soy Broth (Difco Laboratories, Detroit, MI, USA) containing glycerol $15 \% \mathrm{v} / \mathrm{v}$.

\section{PCR analysis}

Integrons were detected by PCR with primers specific for the $5^{\prime}$-conserved segment $\left(5^{\prime}\right.$-CS; 5'-GGCATCC AAGCAGCAAG-3') and the $3^{\prime}$-conserved segment (3'CS; 5'-AAGCAGACTTGACCTGA-3') [11]. The DNA templates for PCR were prepared as described by Bass et al. [12]. The PCR itself was performed in 20- $\mu 1$ volumes containing $200 \mu \mathrm{M}$ dNTP, $1 \mathrm{U}$ Taq DNA polymerase, $10 \times$ PCR buffer (TaKaRa, Shiga, Japan), $0.3 \mu \mathrm{M}$ of each primer and template DNA in a Gene Amp 9700 PCR system (Perkin-Elmer, Norwalk, CT, USA). The PCR conditions consisted of denaturation at $95^{\circ} \mathrm{C}$ for $1 \mathrm{~min}$, then 30 cycles of denaturation for $30 \mathrm{~s}$ at $95^{\circ} \mathrm{C}$, annealing for $30 \mathrm{~s}$ at $55^{\circ} \mathrm{C}$ and extension for $1.5-2 \mathrm{~min}$ at $72^{\circ} \mathrm{C}$. The PCR products were visualised after electrophoresis through an agarose $1.5-2 \%$ gel containing ethidium bromide. Plasmid pUB2401, which harbours transposon Tn21 containing the In2 class 1 integron [2-4], was used as a positive control. Plasmid ColE1::Tn7, which harbours transposon Tn7 with a class 2 integron [2-4], was included as a negative control.

\section{Southern hybridisation}

Plasmid DNA was extracted by the method of Kado and Liu [13], subjected to electrophoresis through an agarose $\quad 0.8 \%$ gel (Boehringer-Mannheim $\mathrm{GmbH}$, Mannheim, Germany), stained with ethidium bromide and photographed under UV transillumination. The gels were then partially depurinated in $0.25 \mathrm{M} \mathrm{HCl}$ and denatured in $0.5 \mathrm{M} \mathrm{NaOH}$, after which the DNA was transferred to a nylon membrane. This was prehybridised, then hybridised with an oligonucleotide probe (5'-GCCTCGACTTCGCTGCTGCCC-3') specific for the intIl gene [14]. This probe was labelled with DIG-ddUTP. A positive hybridisation result was detected with a DIG Luminescent Detection Kit (Boehringer-Mannheim), used according to the manufacturer's recommendations. Luminescence was documented by exposure to Kodak BioMax Light film (Kodak, Rochester, NY, USA).

\section{DNA sequencing and computer analysis of sequence data}

The genetic material inserted within the integronvariable regions was identified by direct nucleotide sequencing. The template amplicons were purified with a QIAquick PCR Purification Kit (QIAGEN GmbH, Hilden, Germany). When two amplicons of different lengths were obtained from a single isolate, each fragment was excised from the agarose gel and purified with a QIAquick Gel Extraction Kit (QIAGEN). Nucleotide sequencing was performed with the ABI PRISM $^{\mathrm{TM}}$ Dye Terminator Cycle Sequencing Ready Reaction Kit (Perkin-Elmer), and the reactions were analysed on an ABI PRISM 377 DNA sequencer. Similarity searches with nucleotide sequences in the GenBank database were performed with the BLASTN program (National Center for Biotechnology Information, USA).

\section{Amplicon cloning}

To further explore the expression of the integron-borne gene cassettes, an amplicon representative of each of the integrons found with different gene cassettes was ligated into the pGEM-T Easy Vector (Promega, Madison, WI, USA) according to the manufacturer's instructions and was then transformed into $E$. coli JM101 [supE thi $\Delta$ (lac-proAB) $\mathrm{F}^{\prime}$ traD36 proAB ${ }^{+}$ lacI $^{\mathrm{q}}$ lacZ $\left.\Delta \mathrm{M} 15\right]$ [15]. Transformants were selected on Luria-Bertani (LB) agar containing ampicillin $100 \mathrm{mg} / \mathrm{L}$, $0.5 \mathrm{mM}$ isopropylthiogalactoside and X-Gal $80 \mu \mathrm{g} / \mathrm{ml}$.

\section{Antimicrobial susceptibility testing}

Antimicrobial susceptibility tests were performed by the disk diffusion method recommended by the National Committee for Clinical Laboratory Standards [16].

\section{Results}

Presence and location of class 1 integrons in E. coli isolates

Class 1 integrons were sought in $104 \mathrm{E}$. coli isolates from clinical specimens by PCR with the 5'-CS and 3'CS primers, which correspond to the sequences flanking the integron-variable regions; 54 (52\%) of the isolates carried detectable class 1 integron structures. The amplicon lengths, corresponding to the approximate sizes of the inserted cassette DNA, varied from 1 to $3 \mathrm{~kb}$ (Table 1 and Fig. 1). Among the 54 isolates, 46 yielded one amplicon and eight yielded two amplicons of different sizes (Table 1 and Fig. 1). None of the $E$. coli isolates had only the integron conserved segments without inserted DNA; if such 'empty' integron structures were present, the resulting amplicons would be $c .150 \mathrm{bp}$ and would have been resolved 
Table 1. Sizes and numbers of amplicons amplified from E. coli isolates with primers $5^{\prime}$-CS and $3^{\prime}-\mathrm{CS}$

\begin{tabular}{lcccc}
\hline & $\begin{array}{c}\text { Isolates yielding single } \\
\text { amplicons }(\mathrm{n}=46)\end{array}$ & & \multicolumn{2}{c}{$\begin{array}{c}\text { Isolates yielding two } \\
\text { amplicons }(\mathrm{n}=8)\end{array}$} \\
\cline { 1 - 2 } $\begin{array}{l}\text { Approximate } \\
\text { length }(\mathrm{kb})\end{array}$ & $\begin{array}{c}\text { Number of } \\
\text { isolates (group) }\end{array}$ & & $\begin{array}{c}\text { Approximate } \\
\text { lengths (kb) }\end{array}$ & $\begin{array}{c}\text { Number of } \\
\text { isolates (group) }\end{array}$ \\
\hline 1.0 & $9(1)$ & $1.0,1.9$ & $3(6)$ \\
1.6 & $6(2)$ & & $1.0,2.1$ & $1(7)$ \\
1.9 & $29(3)$ & & $1.6,1.9$ & $1(8)$ \\
2.0 & $1(4)$ & & $1.6,2.0$ & $3(9)$ \\
3.0 & $1(5)$ & $\ldots$ & $\ldots$ \\
\hline
\end{tabular}

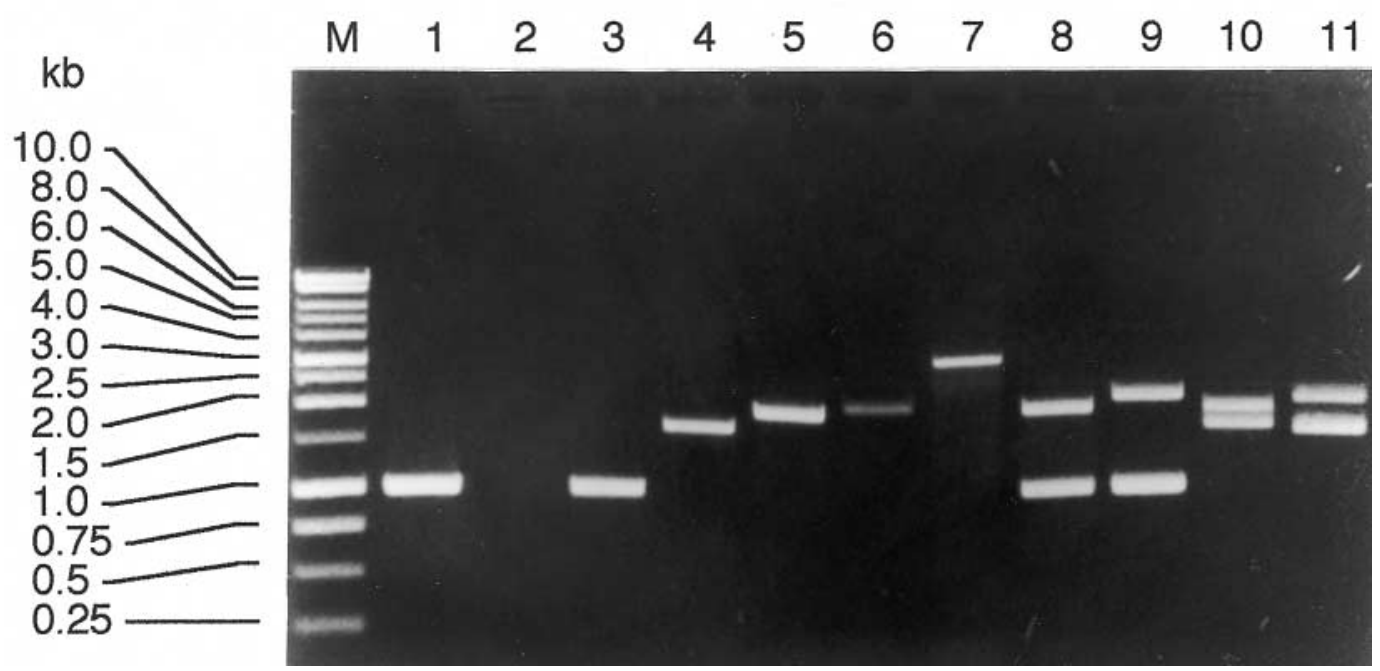

Fig. 1. PCR amplification of the integron-variable regions with the $5^{\prime}$-CS and $3{ }^{\prime}$-CS primers. The amplicons were separated by electrophoresis through an agarose $1.5 \%$ gel. Lane M, size markers; lane 1, Tn21 (positive control); 2 , ColE1::Tn7 (negative control); 3-11, amplicons from clinical E. coli isolates; 3, 1.0-kb amplicon; 4, 1.6-kb amplicon; 5, 1.9-kb amplicon; 6, 2.0-kb amplicon; 7, 3.0-kb amplicon; 8, 1.0-kb and 1.9-kb amplicons; 9, 1.0-kb and 2.1-kb amplicons; 10, $1.6-\mathrm{kb}$ and $1.9-\mathrm{kb}$ amplicons; 11, 1.6-kb and $2.0-\mathrm{kb}$ amplicons.

by the electrophoresis conditions used. The PCR analysis showed that different insert sizes were present, indicating heterogeneity in the gene cassettes; nevertheless, 33 of the 54 isolates with class 1 integrons had $1.9-\mathrm{kb}$ inserts.

Southern hybridisation with an oligonucleotide probe specific for the intIl gene consistently revealed that the integrons were located on plasmids (Fig. 2).

\section{Identification of integron-borne gene cassettes}

Purified PCR products were subjected to DNA sequencing and the gene cassettes thereby identified are listed in Table 2. The integrons were classified into nine groups according to the length and the numbers of amplicons yielded by a single isolate (Table 1). Groups 1 and 2 were further divided into two subgroups, a and $\mathrm{b}$, based on the kinds of gene cassettes identified (Table 2 ). The gene cassettes encoded antibiotic resistance determinants with the possible exception of one open reading frame encoding an unknown product, orfF. These antibiotic resistance gene cassettes included four trimethoprim resistance determinants ( $d f r I a, d f r V, d f r 12$ and $d f r 17)$ and three spectinomycin/streptomycin resistance determinants (aadAla, aadA2 and aadA4).
Other gene cassettes found included $a a d B$, encoding gentamicin resistance; $c m l A$, encoding non-enzymic chloramphenicol resistance [17]; ereA2, encoding erythromycin resistance and blaP1, encoding PSE-1, a class A $\beta$-lactamase [18].

The integrons were found to contain one-to-three gene cassettes and the combinations of these are shown in Table 2. The 1.9-kb amplicon present in 33 isolates carried $d f r 12$, orfF and aadA2 (group 3, 6 and 8). An integron containing two newly described gene cassettes, dfr 17 and aadA4 [19], was found in the five isolates yielding a 1.6-kb amplicon (groups $2 \mathrm{~b}$ and 8 ). The entire sequences of the $d f r 17$ and aadA4 cassettes were deposited in GenBank, under accession no. AF170088. Eight isolates yielded two amplicons of different sizes with primers $5^{\prime}$-CS and $3^{\prime}$-CS; sequencing analysis confirmed the co-existence of two distinct integrons carrying different gene cassettes in these isolates (group 6, 7, 8 and 9).

\section{Antimicrobial resistance in relation to integron carriage}

When isolates contained a gene cassette, the corresponding antibiotic-resistant phenotype was detected 
a

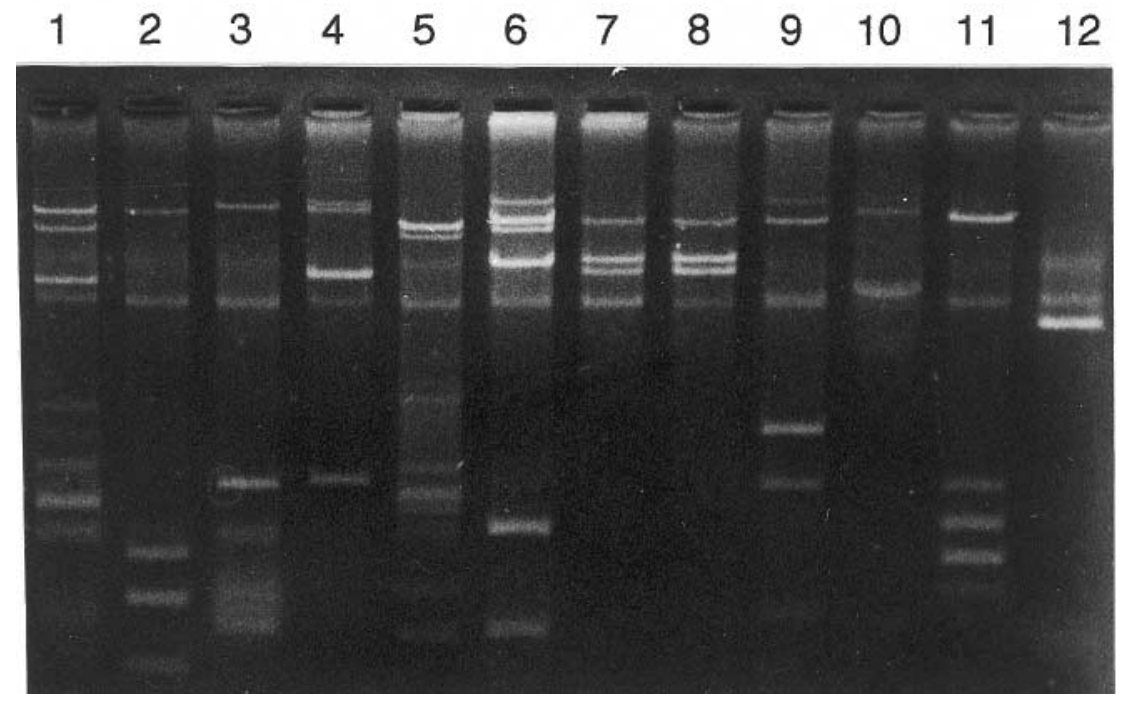

b

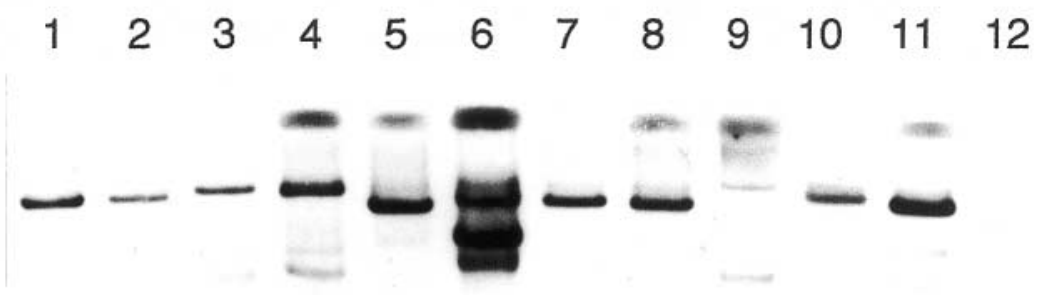

Fig. 2 Agarose gel electrophoresis (a) and Southern hybridisation (b) of plasmid DNA from clinical E. coli isolates. Southern hybridisation was performed with a probe specific for the intI1 gene. Lanes 1-11, E. coli clinical isolates; 12, ColE1::Tn7 (negative control).

consistently (Table 2). Further cloning and expression of the amplicons containing the cassette regions demonstrated that the transformants containing an amplicon expressed phenotypic antibiotic resistance encoded by the gene cassettes present in this amplicon. For instance, the transformants containing the $1.9-\mathrm{kb}$ amplicon, comprising $d f r 12$, orfF and aadA2 cassettes, were resistant to trimethoprim, spectinomycin and streptomycin. Nevertheless, the integron-carried genes did not account for all the phenotypic resistances of the E. coli isolates. Thus, although 39 isolates were resistant to chloramphenicol and 22 were resistant to gentamicin, only one contained an integron carrying the $\mathrm{cmlA}$ and $a a d B$ cassettes (group 5 in Table 2). A few isolates (c. 2-7\%) were resistant to cefmetazole, ceftazidime, moxalactam, aztreonam and netilmicin, drugs to which no integron-associated resistance determinants were found.

\section{Discussion}

Of 104 E. coli isolates, 54 (52\%) had class 1 integron structures, as detected by PCR with primers specific for the CS regions flanking the inserted gene cassettes.
Other reports have shown the prevalence of class 1 integrons in gram-negative clinical isolates to be $c$. $43 \%$ in Western and Central Europe [8], $>50 \%$ in the Netherlands [20] and 59\% in France [7]. Among aminoglycoside-resistant isolates from the USA, nearly $75 \%$ had integrons [11]. These results, together with those obtained in the present study, indicate that class 1 integrons are widespread in clinical isolates. International travel may contribute to their dissemination and that of their host strains. Most $(61 \%)$ of the class 1 integrons found in this study carried $d f r 12$, orf $F$ and aadA2 cassettes. This pattern was also reported in a urinary E. coli isolate from Turku, Finland [21], and in Shigella strains isolated in Finland, but originating from Asia [21]. The present study also revealed an integron carrying two newly characterised gene cassettes, $d$ fr 17 and aadA4. These cassettes were also found in a recent urinary E. coli isolate from Australia [22] and the same aadA cassette (although designated as $\operatorname{aadA5)}$ was found in a class 1 integron in a porcine E. coli isolate from Denmark [23]. These data reveal that the same cassettes can disseminate in different bacterial species, in different human or animal hosts and in different areas of the world. Such observations underscore the concern that human, animal and 
environmental microbial ecosystems are inextricably intertwined, with antibiotic resistance readily crossing ecological boundaries and spreading widely [24]. This makes control much more difficult.

Although the integrons contained different combinations of gene cassettes, some combinations in gene cassettes were frequently conserved, such as dfrIaaadAla and dfr12-orfF-aadA2. This observation may indicate that these gene cassettes have become stably integrated over a long period of time. Although integrase-mediated insertion and excision of gene cassettes has been demonstrated [2-4], Martinez-Freijo et al. also proposed that many inserted gene cassettes may be preserved and stable [6]. Moreover, the transfer of the entire integron, via a plasmid or transposon, is more frequent than single gene mobilisation or integration within the integron [6]. In this study, integrons were located on plasmids, and conjugation experiments revealed that strain-to-strain transfer could occur.

All the 54 isolates with class 1 integrons contained at least one kind of aadA cassette (aadAla, aadA2 or aadA4), alone or in combination with other cassettes. aadA genes confer resistance to spectinomycin and streptomycin and have been found frequently in integrons [6,7]. Moreover, despite minimal use as therapeutic agents, resistances to streptomycin and spectinomycin remain prevalent among $E$. coli isolates $[12,25]$. It seems likely that, due to the lack of direct selection pressure, different aadA variants have not been selected to the extent that has occurred with genes conferring resistance to trimethoprim and $\beta$-lactams. However, the finding of a novel streptomycin/spectinomycin resistance gene indicated that variations in the aadA gene do arise. Moreover, streptomycin is still used extensively in animal husbandry [26], perhaps selecting new aadA gene variants that may spread to pathogenic bacteria isolated from man.

Some researchers propose that the aadA gene may be the first cassette acquired by integrons $[27,28]$. In the present study, most aadA cassettes were combined with other kinds of gene cassettes, adjacent to the 5'conserved segment and thus upstream of the aadA cassettes. To this extent the results could support the suggestion that aadA is the first cassette acquired by integrons, because a gene cassette preferentially recombines into the attI site, rather than into the attC sites at the $3^{\prime}$-ends of cassettes already present in the integron [3]. On the other hand, aadA genes were rarely the sole cassettes in integrons. This finding is different from that of Rosser and Young [28], who found that aadA was often the sole cassette in the variable region of integrons from coliform bacteria, Pseudomonas-like and Vibrio-like isolates from a natural estuarine environment. The disagreement might reflect the fact that clinical isolates are exposed to more antibiotic selection than environmental bacteria, promoting accumulation of multiple gene cassettes. 
Besides $\operatorname{aad} A$, other frequently found cassettes were those encoding resistance to trimethoprim $(d f r)$, which were present in $43(80 \%)$ of the isolates. Four $d f r$ gene types cassettes were identified. All the $54 \mathrm{E}$. coli isolates with class 1 integrons showed resistance to sulphonamides, perhaps reflecting the fact that co-trimoxazole is frequently used to treat $E$. coli infection. Such specific selection pressure may favour the acquisition and maintenance of a trimethoprim resistance cassette by class 1 integrons also containing sull in the $3^{\prime}$ region [23]. This selection pressure also may explain the increasing number of different types of cassette-encoded dihydrofolate reductase. For example, $d f r 17$ is a novel trimethoprim resistance gene cassette. Furthermore, $d f r$ cassettes were located directly behind the 5 '-conserved segment. This site is the closest to the promoter, giving high-level expression and contingent resistance.

Antimicrobial susceptibility tests confirmed that the gene cassettes in the integrons contributed to resistance, as also found by others [7, 28]. Many isolates also had other antibiotic resistances not encoded by integron-associated resistance gene cassettes. These resistances may have resulted from chromosomal mutation, plasmid acquisition or the presence of other integrons besides class 1 types.

The present study revealed that class 1 integrons are widespread in clinical isolates. Their location on plasmids may contribute to horizontal dissemination of the antibiotic resistance gene cassettes. All the integrons contained antibiotic resistance gene cassettes. The study of integrons and their associated gene cassettes could provide information about which antibiotics should be used most carefully to prevent further accumulation of resistance.

This study was supported by grants from the Department of Health, ROC (DOH88-TD-1009 and DOH89-TD-1005) and partially from the Kaohsiung Medical University (M8725).

\section{References}

1. Stokes HW, Hall RM. A novel family of potentially mobile DNA elements encoding site-specific gene-integration functions: integrons. Mol Microbiol 1989; 3: 1669-1683.

2. Hall RM, Stokes HW. Integrons: novel DNA elements which capture genes by site-specific recombination. Genetica 1993; 90: $115-132$.

3. Hall RM, Collis CM. Mobile gene cassettes and integrons: capture and spread of genes by site-specific recombination. Mol Microbiol 1995; 15: 593-600.

4. Recchia GD, Hall RM. Gene cassettes: a new class of mobile element. Microbiology 1995; 141: 3015-3027.

5. Hall RM, Brookes DE, Stokes HW. Site-specific insertion of genes into integrons: role of the 59-base element and determination of the recombination cross-over point. $\mathrm{Mol}$ Microbiol 1991; 5: 1941-1959.

6. Martinez-Freijo P, Fluit AC, Schmitz F-J, Verhoef J, Jones ME. Many class I integrons comprise distinct stable structures occurring in different species of Enterobacteriaceae isolated from widespread geographic regions in Europe. Antimicrob Agents Chemother 1999; 43: 686-689.

7. Sallen B, Rajoharison A, Desvarenne S, Mabilat C. Molecular epidemiology of integron-associated antibiotic resistance genes in clinical isolates of Enterobacteriaceae. Microb Drug Resist 1995; 1: 195-202.

8. Martinez-Freijo P, Fluit AC, Schmitz F-J, Grek VSC, Verhoef $\mathrm{J}$, Jones ME. Class I integrons in Gram-negative isolates from different European hospitals and association with decreased susceptibility to multiple antibiotic compounds. J Antimicrob Chemother 1998; 42: 689-696.

9. Gonzalez G, Sossa K, Bello H, Dominguez M, Mella S, Zemelman R. Presence of integrons in isolates of different biotypes of Acinetobacter baumannii from Chilean hospitals. FEMS Microbiol Lett 1998; 161: 125-128.

10. Chang SC, Hsieh WC. Current status of bacterial antibiotic resistance in Taiwan. J Infect Dis Soc ROC 1996; 7: 83-88.

11. Lévesque $\mathrm{C}$, Piché L, Larose C, Roy PH. PCR mapping of integrons reveals several novel combinations of resistance genes. Antimicrob Agents Chemother 1995; 39: 185-191.

12. Bass L, Liebert $\mathrm{CA}$, Lee $\mathrm{MD}$ et al. Incidence and characterization of integrons, genetic elements mediating multiple-drug resistance, in avian Escherichia coli. Antimicrob Agents Chemother 1999; 43: 2925-2929.

13. Kado CI, Liu S-T. Rapid procedure for detection and isolation of large and small plasmids. $J$ Bacteriol 1981; 145: 1365-1373.

14. Schwocho LR, Schaffner CP, Miller GH, Hare RS, Shaw KJ. Cloning and characterization of a 3-N-aminoglycoside acetyltransferase gene, aac(3)-Ib, from Pseudomonas aeruginosa. Antimicrob Agents Chemother 1995; 39: 1790-1796.

15. Yanisch-Perron C, Vieira J, Messing J. Improved M13 phage cloning vectors and host strains: nucleotide sequences of the M13mp18 and pUC19 vectors. Gene 1985; 33: 103-119.

16. National Committee for Clinical Laboratory Standards. Performance standards for antimicrobial disk susceptibility test, 5th edn. Approved Standard: M2-A5. Villanova, PA, NCCLS. 1993.

17. Bissonnette L, Champetier S, Buisson J-P, Roy PH. Characterization of the nonenzymatic chloramphenicol resistance $(\mathrm{cmlA})$ gene of the In4 integron of Tn1696: similarity of the product to transmembrane transport proteins. J Bacteriol 1991; 173: 4493-4502.

18. Huovinen P, Jacoby GA. Sequence of the PSE-1 $\beta$ lactamase gene. Antimicrob Agents Chemother 1991; 35: 2428-2430.

19. Chang C-Y, Chang L-L, Chang Y-H, Lee T-M, Li Y-H, Chang S-F. Two new gene cassettes, dfrl7 (for trimethoprim resistance) and aadA4 (for spectinomycin/streptomycin resistance), inserted in an Escherichia coli class 1 integron. $J$ Antimicrob Chemother 2000 (in press).

20. Jones ME, Peters E, Weersink A-M, Fluit A, Verhoef J. Widespread occurrence of integrons causing multiple antibiotic resistance in bacteria. Lancet 1997; 349: 1742-1743.

21. Heikkilä E, Skurnik M, Sundström L, Huovinen P. A novel dihydrofolate reductase cassette inserted in an integron borne on a Tn21-like element. Antimicrob Agents Chemother 1993; 37: $1297-1304$.

22. White PA, McIver CJ, Deng Y-M, Rawlinson WD. Characterisation of two new gene cassettes, aadA5 and dfrA17. FEMS Microbiol Lett 2000; 182: 265-269.

23. Sandvang D. Novel streptomycin and spectinomycin resistance gene as a gene cassette within a class 1 integron isolated from Escherichia coli. Antimicrob Agents Chemother 1999; 43: 3036-3038.

24. Witte W. Medical consequences of antibiotic use in agriculture. Science 1998; 279: 996-997.

25. Chiew Y-F, Yeo S-F, Hall LMC, Livermore DM. Can susceptibility to an antimicrobial be restored by halting its use? The case of streptomycin versus Enterobacteriaceae. $J$ Antimicrob Chemother 1998; 41: 247-251.

26. Liebert CA, Hall RM, Summers AO. Transposon $\operatorname{Tn} 21$, flagship of the floating genome. Microbiol Mol Biol Rev 1999; 63: 507-522.

27. Bissonnette I, Roy PH. Characterization of In0 of Pseudomonas aeruginosa plasmid pVS1, an ancestor of integrons of multiresistance plasmids and transposons of gram-negative bacteria. J Bacteriol 1992; 174: 1248-1257.

28. Rosser SJ, Young H-K. Identification and characterization of class I integrons in bacteria from an aquatic environment. $J$ Antimicrob Chemother 1999; 44: 11-18. 